Mathematical Research Letters 6, 183-194 (1999)

\title{
POLYCYCLIC-BY-FINITE GROUP ALGEBRAS ARE CATENARY
}

\author{
EDWARd S. LetzTer AND MARTin LoREnZ
}

\begin{abstract}
We show that group algebras $k G$ of polycyclic-by-finite groups $G$, where $k$ is a field, are catenary: If $P=I_{0} \subsetneq I_{1} \subsetneq \cdots \subsetneq I_{m}=P^{\prime}$ and $P=J_{0} \subsetneq$ $J_{2} \subsetneq \cdots \subsetneq J_{n}=P^{\prime}$ are both saturated chains of prime ideals of $k G$, then $m=n$.
\end{abstract}

\section{Introduction}

Group algebras $k G$ of polycyclic-by-finite groups $G$, over a field $k$, are among the most prominent special classes of noncommutative noetherian rings, partly because the only known noetherian group algebras (over fields) are of the form $k G$, and partly because of the intrinsic group theoretic interest in $G$. Indeed, the investigation of the prime ideals of $k G$ was a major enterprise throughout the 1970's. Zalesskii's work [20] initiated a program that was essentially completed in 1980 by [11], [10] and [1]; the most notable contribution was by Roseblade [15]. The aforementioned papers resulted in a detailed, albeit complicated, description of the prime ideals of $k G$, and the subject has been largely dormant ever since. Disappointingly, the question of whether $k G$ is, or is not, catenary (see below) remained unanswered, despite several attempts in that direction: [17] gave an affirmative answer for f.g. nilpotent groups, [15, Corrigenda] did the same for $\mathfrak{Z}$ groups, and [10] settled the case of orbitally sound-by-(finite nilpotent) groups. But the intervening almost 20 years have witnessed substantial progress in our understanding of prime ideals in both noetherian rings and their extensions (see, e.g., [4]), and so we can now offer in the present paper a proof of the following

Theorem. Group algebras of polycyclic-by-finite groups are catenary.

Recall that a ring $R$ is called catenary when the following holds: For every inclusion $P \subsetneq P^{\prime}$ of prime ideals in $R$, if $P=I_{0} \subsetneq I_{1} \subsetneq \cdots \subsetneq I_{m}=P^{\prime}$ and $P=J_{0} \subsetneq J_{2} \subsetneq \cdots \subsetneq J_{n}=P^{\prime}$ are both chains of prime ideals that cannot be lengthened by the insertion of extra primes, then $m=n$. A fundamental result of commutative algebra asserts that all affine commutative $k$-algebras are catenary. Various noncommutative generalizations of this result are known: Affine PI-algebras [16], enveloping algebras of finite dimensional solvable Lie

Received January 21, 1999.

Research of the first author supported in part by NSF Grant DMS-9623579

Research of the second author supported in part by NSF Grant DMS-9618521

Key words and phrases. group algebra, polycyclic-by-finite group, prime ideal, catenary ring, noetherian ring, second layer condition

1991 Mathematics Subject Classisfication. 16S34, 16D30, 16P40 
(super-)algebras [2], [8] and, most recently, certain algebras arising from quantum groups [3] have all been proven to be catenary.

Notations and Conventions. Throughout this note, $k$ will denote a commutative field. Furthermore, $\subsetneq$ denotes strict inclusion, while equality is permitted in $\subset$; similarly for $\supsetneq$ and $\supset$. We will write ann $X_{R}$ when referring to the right annihilator of $X$ in $R$ and $\operatorname{ann}_{S} Y$ when referring to the left annihilator of $Y$ in $S$.

\section{Preliminaries}

1.1. Catenarity. A ring $R$ is called catenary if, given prime ideals $Q \subsetneq Q^{\prime}$ of $R$, any two saturated chains of primes between $Q$ and $Q^{\prime}$ have the same length. Here, a chain $Q_{0} \subsetneq Q_{1} \subsetneq \cdots \subsetneq Q_{m}$ of prime ideals of $R$ is saturated if $Q_{i-1}$ and $Q_{i}$ are adjacent for all $1 \leq i \leq m$; a pair of prime ideals $Q$ and $Q^{\prime}$ in $R$ are adjacent if $Q \subsetneq Q^{\prime}$ and if there exist no prime ideals $Q^{\prime \prime}$ of $R$ such that $Q \subsetneq Q^{\prime \prime} \subsetneq Q^{\prime}$.

Recall that the height of a prime ideal $P$ of $R$ is the supremum of the lengths $n$ of all chains $P=P_{0} \supsetneq P_{1} \supsetneq \cdots \supsetneq P_{n}$ of prime ideals descending from $P$. In all known noetherian rings the prime ideals have finite height; for group algebras of polycyclic-by-finite groups, see [15, Theorem H1]. Assuming finiteness of heights, $R$ is certainly catenary if the following condition is satisfied:

height $P^{\prime}=$ height $P+1$ holds whenever $P \subsetneq P^{\prime}$ are adjacent prime ideals.

If every prime of $R$ contains exactly one minimal prime, then (1) is actually equivalent with catenarity.

For group algebras $k G$ of polycyclic-by-finite groups $G$, the latter condition on minimal primes holds. Indeed, if $F$ denotes the finite radical of $G$, that is, the largest finite normal subgroup of $G$, then the unique minimal prime of $k G$ contained in a given prime ideal $P$ of $k G$ is $(P \cap k F) k G$; see [15, Corollary 22]. Thus, our objective will be to verify (1) for $k G$.

1.2. Heights. Let $k G$ be the group algebra of a polycyclic-by-finite group $G$. Following Roseblade [15] we put, for any ideal $I$ of $k G$,

$$
I^{\dagger}=G \cap(1+I) \text {. }
$$

Thus $I^{\dagger}$ is the kernel of the natural homomorphism of $G$ into the group of units of $k G / I$, and $I$ contains the kernel of the natural homomorphism $k G \rightarrow k\left[G / I^{\dagger}\right]$. The ideal $I$ is said to be faithful iff $I^{\dagger}=1$. The image of $I$ in $k\left[G / I^{\dagger}\right]$ is always faithful.

The following Lemma will be used to reduce the proof of (1) to the case where $P$ is faithful. Its essence is in $[15,2.4]$, at least for Roseblade's $\mathfrak{Z}$-groups; the general case follows rather routinely. The term

$$
p_{G}(N)
$$


in the formula below denotes the $G$-plinth length of the normal subgroup $N$ of $G$. For the definition and basic properties of plinth lengths, the reader is referred to $[15,2.3]$. We remark that $[15,2.3]$ assumes $N$ to be polycyclic, but the notion of plinth length is insensitive to finite factors and applies to polycyclic-by-finite groups as well.

Lemma. Let $k G$ be the group algebra of a polycyclic-by-finite group $G$, let $P$ be a prime ideal of $k G$, and let $N$ be a normal subgroup of $G$ that is contained in $P^{\dagger}$. Then

$$
\text { height } P=\text { height } \bar{P}+p_{G}(N) \text {, }
$$

where $\bar{P}$ denotes the image of $P$ in $k[G / N]$.

Proof. Let $G_{0}$ denote a normal $\mathfrak{Z}$-group of finite index in $G$ (see $[15,1.3]$ ) and put $N_{0}=N \cap G_{0}$. Further, write

$$
P \cap k G_{0}=\bigcap_{g \in G} P_{0}^{g}
$$

for some prime ideal $P_{0}$ of $k G_{0}$; cf. [15, 4.1] or [11, Lemmas 1.3(i), 1.4(i)]. Then $P^{\dagger} \cap G_{0} \subset P_{0}^{\dagger}$, and so $N_{0} \subset P_{0}^{\dagger}$. Letting - denote images in $k[G / N]$, it follows that $\bar{P} \cap k\left[\overline{G_{0}}\right]=\bigcap_{x \in \bar{G}}{\overline{P_{0}}}^{x}$ and $\overline{P_{0}}$ is prime in $k\left[\overline{G_{0}}\right]$. Thus, by $[15,8.1]$ or [11, Lemma 2.5(i)], height $P=$ height $P_{0}$ and height $\bar{P}=$ height $\overline{P_{0}}$. Finally, $p_{G}(N)=p_{G_{0}}\left(N_{0}\right)$, since $G_{0}$ has finite index in $G$. Therefore, replacing $G$ by $G_{0}$ etc., we may assume that $G$ is a $\mathfrak{Z}$-group. In this case, the desired formula follows from Roseblade's height formula in $[15,2.4]$, noting that Roseblade's $\lambda$ equals height for $\mathfrak{Z}$-groups and that $p_{G}($.$) is additive on short exact sequences$ of $G$-groups.

1.3. Second layer theory. We review some relevant results and background information from noetherian ring theory.

(i) Definitions of the right and left strong second layer conditions of Jategaonkar may be found in [4, p. 183]; also see [5]. When we refer to noetherian rings having the strong second layer condition, abbreviated sslc, we mean rings satisfying both the right and left strong second layer conditions.

(ii) Group rings of polycyclic-by-finite groups over commutative noetherian coefficient rings satisfy the sslc; see [5, A.4.6].

(iii) Let $U$ and $V$ be noetherian rings satisfying the sslc. Suppose that $B$ is a $U$ - $V$-bimodule finitely generated and faithful on each side. It was proved by Jategaonkar that $U$ and $V$ have the same classical Krull dimension; see, for example, $[4,12.5]$ or $[5,8.2 .8]$. We will use $\operatorname{clKdim}($.$) to denote classical Krull$ dimension.

(iv) Let $U$ be a prime noetherian ring, and let $M$ be a nonzero left $U$-module. Recall that $u \in U$ is regular provided $u v$ and $v u$ are nonzero for all nonzero $v \in U$. Further recall that $M$ is torsion free provided $u . m \neq 0$ for every regular element $u \in U$ and every $0 \neq m \in M$. It follows from Goldie's Theorem, when 
${ }_{U} M$ is torsion free, that every nonzero submodule of $M$ is faithful. If $N$ is a $U$-module for which every nonzero submodule is faithful, then we say that $N$ is fully faithful over $U$.

(v) Let $P$ and $Q$ be prime ideals in a noetherian $\operatorname{ring} U$ satisfying the sslc. We say that $P$ is linked to $Q$, and write $P \rightsquigarrow Q$, if there exists a $U$ - $U$-bimodule factor $B$ of $(P \cap Q) / P Q$ with the following properties: $\operatorname{ann}_{U} B=P$, ann $B_{U}=Q$, and $B$ is torsion free on each side as a $U / P-U / Q$-bimodule. A graph structure is thus imposed on the set of prime ideals, and if $X$ is a subset thereof then the link closure of $X$ will be defined to be the union of the connected components of the prime ideals in $X$. Note, by (iii), that if $P \rightsquigarrow Q$ then $\operatorname{clKdim}(U / P)=$ $\operatorname{clKdim}(U / Q)$.

(vi) Continue to let $U$ be a noetherian ring satisfying the sslc, and let $M$ be a finitely generated left $U$-module. A prime ideal $P$ of $U$ is said to be an associated prime of $M$ if there exists a submodule $N$ of $M$ such that $\operatorname{ann}_{U} N=P$ and such that $N$ is a fully faithful $U / P$-module. By noetherianity, the set of associated primes of $M$ is not empty. Also, if $M^{\prime}$ is an essential extension of $M$, then the sets of associated primes of $M$ and $M^{\prime}$ are the same. Let $X$ denote the link closure of the set of associated primes of $M$. In [4, 11.4], for example, it is proved that $M$ is annihilated by a (finite) product of prime ideals in $X$. (This last assertion follows from an iterated application of the Main Lemma of Jategaonkar; see [5].)

1.4. Krull dimension. Here we collect some facts on Krull dimension, in the sense of Gabriel and Rentschler, that will be needed later. The Krull dimension of a module will be denoted $\operatorname{Kdim}(M)$; for the definition and basic facts concerning Kdim, we refer the reader to [4, Chapter 13]. All rings and modules discussed below are assumed noetherian, and so $\operatorname{Kdim}(M)$ will always exist. Further, $\operatorname{Kdim}(U)$, for a ring $U$, will stand for $\operatorname{Kdim}\left(U_{U}\right)$.

(i) A nonzero module $M$ is called homogeneous (or $\alpha$-homogeneous, if $\operatorname{Kdim}(M)=\alpha$ ) provided all nonzero submodules of $M$ have the same Krull dimension as $M$. Prime rings are homogeneous; see [4, p. 229]. Further, finite subdirect products and extensions of $\alpha$-homogeneous modules are easily seen to be $\alpha$-homogeneous.

(ii) Assume the ring $U$ is (right) homogeneous. If the element $u \in U$ satisfies $\operatorname{Kdim}(U / u U)<\operatorname{Kdim}(U)$ then $u$ is regular. This assertion can be proved as follows: First, the left regularity of $u$ follows from [14, 6.8.12]. As to right regularity (which is due to Stafford [19]), observe that $(U / u U)_{U}$ maps onto $\left(u^{n-1} U / u^{n} U\right)_{U}$ for all $n>0$. Hence, $\operatorname{Kdim}\left(u^{n-1} U / u^{n} U\right)_{U} \leq \operatorname{Kdim}(U / u U)_{U}<\operatorname{Kdim}(U)$ and, consequently, $\operatorname{Kdim}\left(U / u^{n} U\right)_{U}<\operatorname{Kdim}(U)$. But, by "Fitting's Lemma" [14, 2.3.2(ii)], there is an $n>0$ such that the right annihilator, $\operatorname{ann}\left(u^{n}\right)_{U}$, of $u^{n}$ in $U$ embeds into $U / u^{n} U$. Therefore, $\operatorname{Kdim}\left(\operatorname{ann}\left(u^{n}\right)_{U}\right)<\operatorname{Kdim}(U)$, whence $\operatorname{ann}\left(u^{n}\right)_{U}=0$, by homogeneity. This proves that $u$ is right regular.

(iii) For group algebras $k G$ of polycyclic-by-finite groups $G$, one has $\operatorname{Kdim}(k G)=$ 
$h(G)$, the Hirsch number of $G$; see [18]. If $H$ is a subgroup of finite index in $G$, then for any (right) $k G$-module $M$, one has

$$
\operatorname{Kdim}\left(M_{k G}\right)=\operatorname{Kdim}\left(M_{k H}\right) .
$$

If $H$ is normal, this is covered by a more general result on finite normalizing extensions; see [7, Théorème 5.3]. The general case follows by passing to the normal core $H_{G}=\bigcap_{g \in G} H^{g}$, a normal subgroup of finite index in $G$ (and in $H$ ).

(iv) Let $G, H$, and $M$ be as above and suppose that $M=W k G$ for some submodule $W$ of $M_{k H}$. Then

$$
\operatorname{Kdim}(M)=\operatorname{Kdim}(W) .
$$

Indeed, by (iii), it suffices to prove $\operatorname{Kdim}\left(M_{k N}\right)=\operatorname{Kdim}\left(W_{k N}\right)$, where $N=H_{G}$ is the normal core of $H$. But, as $k N$-module, $M=W k G$ is a finite sum of $G$ conjugates of $W_{k N}$ all of which have isomorphic submodule lattices, and hence the same $\operatorname{Kdim}$. Thus, $\operatorname{Kdim}\left(M_{k N}\right)=\operatorname{Kdim}\left(W_{k N}\right)$, as required.

(v) Keeping the above notations $G$ and $H$, a $k G$-module $M$ is $\alpha$-homogeneous if and only if it is so as $k H$-module. To verify this claim, first note that $\alpha$ homogeneity as $k H$-module certainly entails $\alpha$-homogeneity over $k G$, by (iii). Conversely, if $M_{k G}$ is $\alpha$-homogeneous and $W$ is a nonzero submodule of $M_{k H}$, then $W k G$ is a nonzero $k G$-submodule of $M$, and so $\alpha=\operatorname{Kdim}(W k G)=$ $\operatorname{Kdim}(W)$, by (iv). Finally, if $M=W k G$ for some submodule $W$ of $M_{k H}$, then $M$ is $\alpha$-homogeneous if and only if $W$ is. Indeed, if $M=W k G$ is $\alpha$-homogeneous and $X$ is a nonzero submodule of $W$, then $\alpha=\operatorname{Kdim}(X k G)=\operatorname{Kdim}(X)$, by (iv). Conversely, assume that $W$ is $\alpha$-homogeneous. It suffices to show that $M=W k G$ is $\alpha$-homogeneous as $k N$-module, where $N=H_{G}$. But $M_{k N}$ is a finite sum of $G$-conjugates of $W_{k N}$, and all these are $\alpha$-homogeneous. Hence $M_{k N}$ is $\alpha$-homogeneous.

\section{Induced ideals}

Throughout this section, $R \subset S$ is an extension of rings. Additional assumptions will be required later.

2.1. Definition and basic properties. For any ideal $I$ of $R$, we put

$$
I^{S}=\operatorname{ann}(S / I S)_{S}=\{s \in S \mid S s \subset I S\} .
$$

In other words, $I^{S}$ is the largest ideal of $S$ that is contained in $I S$. We refer to $I^{S}$ as the induced ideal of $I$, and we will say that ideals in $S$ of this general form are induced from $R$. In the case of group algebras $R=k H \subset S=k G$, for some subgroup $H$ of $G$, one also writes $I^{G}$ in place of $I^{S}$ and calls these ideals induced from $H$.

The induction operator

$$
(.)^{S}: \text { Ideals of } R \rightarrow \text { Ideals of } S,
$$


obviously preserves inclusions. Furthermore,

$$
I_{1}^{S} I_{2}^{S} \subset\left(I_{1} I_{2}\right)^{S},
$$

holds for any two ideals $I_{1}, I_{2}$ of $R$. Indeed, $I_{1}^{S} I_{2}^{S} \subset I_{1} S I_{2}^{S} \subset I_{1} I_{2} S$.

2.2. Minimal covering primes. Equation (3) has the following consequence.

Lemma. Let $P$ be a prime ideal of $S$, and let $Q$ be an ideal of $R$ maximal among ideals whose induced ideals are contained in $P$. Then $Q$ is prime.

Proof. Let $I_{1}$ and $I_{2}$ be ideals of $R$ properly containing $Q$. Then $I_{1}^{S}$ and $I_{2}^{S}$ are not contained in $P$, and hence neither is their product. By $(3),\left(I_{1} I_{2}\right)^{S}$ is not contained in $P$, whence $I_{1} I_{2}$ is not contained in $Q$.

In the situation of the above Lemma, $P$ will often actually be minimal over $Q^{S}$. A sufficient condition for this to happen is given in the following:

Proposition. Suppose that $S$ is finitely generated and free as a left $R$-module. Assume further that $R$ and $S$ are noetherian and satisfy the sslc. Let $Q$ be a prime ideal of $R$, and let $P$ be a prime ideal of $S$ containing $Q^{S}$. Then the following are equivalent:

i. $P$ is minimal over $Q^{S}$;

ii. $S / P$ and $R / Q$ have the same classical Krull dimension;

iii. $Q$ is maximal among ideals in $R$ whose induced ideal is contained in $P$.

Proof. Set $M=S / Q S$ and $J=Q^{S}=\operatorname{ann} M_{S}$. By hypothesis on ${ }_{R} S, M$ is free of finite rank as left $R / Q$-module. In particular, it follows from (1.3 iii) that $\operatorname{clKdim}(R / Q)=\operatorname{clKdim}(S / J)$, and $\operatorname{so} \operatorname{clKdim}(S / P) \leq \operatorname{clKdim}(R / Q)$.

( $\mathrm{i} \Rightarrow \mathrm{ii})$ Note that $M$ is nonzero and torsion free as a left $R / Q$-module. Therefore, by $[4,7.7]$, there exist prime ideals $P_{1}, \ldots, P_{m}$ of $S$, and a series of $R$ - $S$-bimodules

$$
0=M_{0} \subsetneq M_{1} \subsetneq \cdots \subsetneq M_{m}=M
$$

with the following properties: For each $1 \leq i \leq n$, the right annihilator in $S$ of $M_{i} / M_{i-1}$ is equal to $P_{i}$, and $M_{i} / M_{i-1}$ is faithful on each side as an $R / Q-S / P_{i^{-}}$ bimodule. Note that all of the prime ideals in $S$ minimal over $J$, and in particular $P$, are included among $P_{1}, \ldots, P_{m}$. Therefore, $\operatorname{clKdim}(S / P)=\operatorname{clKdim}(R / Q)$, by (1.3 iii).

(ii $\Rightarrow$ i) If $P$ is not minimal over $J$, then

$$
\operatorname{clKdim}(S / P)<\operatorname{clKdim}(S / J)=\operatorname{clKdim}(R / Q) .
$$

(ii $\Rightarrow$ iii) Suppose that $I$ is an ideal of $R$, properly containing $Q$, such that $I^{S} \subset P$. Since $S$ is free as a left $R$-module, $\operatorname{ann}_{R}(S / I S)=I$. Therefore, by (1.3 iii),

$$
\operatorname{clKdim}(R / Q)>\operatorname{clKdim}(R / I)=\operatorname{clKdim}\left(S / I^{S}\right) \geq \operatorname{clKdim}(S / P) .
$$


(iii $\Rightarrow$ ii) $\operatorname{Suppose~that~} \operatorname{clKdim}(S / P) \neq \operatorname{clKdim}(R / Q)$, and so $\operatorname{clKdim}(S / P)<$ $\operatorname{clKdim}(R / Q)$. We will show that there exists an ideal $I$ of $R$, strictly containing $Q$, such that $I^{S} \subset P$.

By $[4,12.3]$, there exists a series of $R$-S-bimodules

$$
0 \subset L_{1} \subsetneq L_{2} \subset M
$$

such that $\operatorname{ann}\left(L_{2} / L_{1}\right)_{S}=P$, and such that $L_{2} / L_{1}$ is torsion free as a right $S / P$ module. Let $K$ be an $R$-S-sub-bimodule of $M$, containing $L_{1}$, and maximal with respect to $K \cap L_{2} \subset L_{1}$. Set $N=M / K$. Note that $L_{2} / L_{1}$ embeds as an $R$-Sbimodule into $N$; we will let $L$ denote the image of this embedding. Observe that every nonzero $R$-S-sub-bimodule of $N$ intersects $L$ nontrivially. Also, by (1.3 iv), $L$ is a fully faithful right $S / P$-module.

Let $Q^{\prime}$ be an associated prime of ${ }_{R} L$, and let $L^{\prime}=\operatorname{ann} Q_{L}^{\prime}$. Note that $L^{\prime}$ is a nonzero $R$-S-sub-bimodule of $L$, and that $Q^{\prime}=\operatorname{ann}_{R} L^{\prime}$. In particular, $L^{\prime}$ is an $R / Q^{\prime}-S / P$-bimodule finitely generated and faithful on each side. Consequently, by $(1.3$ iii $), \operatorname{clK} \operatorname{dim}\left(R / Q^{\prime}\right)=\operatorname{clKdim}(S / P)$. Now let $X$ denote the link closure of the associated primes of ${ }_{R} L$. By $(1.3 \mathrm{v})$, if $Q^{\prime \prime} \in X$, then $\operatorname{clKdim}\left(R / Q^{\prime \prime}\right)=$ $\operatorname{clKdim}(S / P)<\operatorname{clKdim}(R / Q)$.

Now let $T$ be a left $R$-submodule of $N$ maximal with respect to $T \cap L=0$. Set $V=N / T$ and $I=\operatorname{ann}_{R} V$. Note that $I \supset Q$ and that $L$ embeds into ${ }_{R} V$ as an essential submodule. It now follows from $(1.3 \mathrm{vi})$ that $V$ is annihilated on the left by a product $Q_{1} \cdots Q_{n}$ of prime ideals $Q_{1}, \ldots, Q_{n} \in X$. However, the classical Krull codimension of each $Q_{i}$ is strictly smaller than $\operatorname{clKdim}(R / Q)$, and so $I$ properly contains $Q$.

Since $I V=0$, we have $I N \subset T$, and so $I N \cap L=0$. However, $I N$ is an $R-S$ sub-bimodule of $N$, and so $I N=0$ because each nonzero $R$-S-sub-bimodule of $N$ intersects $L$ nontrivially. Therefore, $I M \subset K$, and so $I M \cap L_{2} \subset L_{1}$. Hence, $L_{2} / L_{1}$ is an $R$-S-bimodule subfactor of $M / I M$. Since $\operatorname{ann}\left(L_{2} / L_{1}\right)_{S}=P$ and $M / I M \cong S / I S$, we conclude that

$$
P \supset \operatorname{ann}(M / I M)_{S}=\operatorname{ann}(S / I S)_{S}=I^{S} .
$$

The proposition follows.

Remark. The argument establishing (iii $\Rightarrow$ ii) above is similar to the proof in $[9,4.6]$ of "lying over" for finite extensions of noetherian rings with the second layer condition.

2.3. The case of group rings. We will broaden the list of equivalent properties in Proposition 2.2 by adding another condition concerning heights. This strengthening of the proposition seems, however, to require further restrictions on the ring embedding $R \subset S$. Therefore, we will concentrate here on extensions of group algebras,

$$
R=k H \subset S=k G,
$$

where $G$ is a polycyclic-by-finite group and $H$ is a subgroup of finite index in $G$. 
We start with a lemma on (Krull) homogeneity whose essence is gleaned from [1, Lemma 4.2].

Lemma. Let $G$ be a polycyclic-by-finite group, let $H$ be a subgroup of finite index in $G$, and put $N=H_{G}$, the normal core of $H$. Further, let $Q$ be a prime ideal of $k H$ and put $I=Q^{G} \cap k N$. Then

$$
k H / Q, k G / Q^{G} \text {, and } k G / I k G
$$

are all (right) $\alpha$-homogeneous rings, where $\alpha=\operatorname{Kdim}(k H / Q)$.

Proof. First, $k H / Q$ is prime and therefore $\alpha$-homogeneous (1.4i). Next, by [11, Lemma 1.2(i)],

$$
Q^{G}=\bigcap_{g \in G} Q^{g} k G
$$

and this intersection is finite. Thus, as right $k G$-module, $k G / Q^{G}$ is a finite subdirect product of the modules $k G / Q^{g} k G$. Furthermore, each $k G / Q^{g} k G$ is generated, as $k G$-module, by the $k\left[H^{g}\right]$-submodule $k\left[H^{g}\right] / Q^{g}$. Now $k\left[H^{g}\right] / Q^{g}$ is isomorphic to $k H / Q$ (as ring) and is therefore $\alpha$-homogeneous. Consequently, each $k G / Q^{g} k G$ is $\alpha$-homogeneous, by $(1.4 \mathrm{v})$, and hence so is $k G / Q^{G}$, by (1.4 i).

Finally, using (4), $I=\bigcap_{g \in G}(Q \cap k N)^{g}$ and, by [1, Lemma 4.2(i)], $k N / Q \cap k N$ is $\alpha$-homogeneous. Therefore, so are its $G$-conjugates $k N /(Q \cap k N)^{g}$ and consequently $k N / I$ as well, by the usual (finite) subdirect product argument (1.4i). Since $k N / I$ generates the $k G$-module $k G / I k G$, the latter is $\alpha$-homogeneous, by $(1.4 \mathrm{v})$, and the proof is complete.

Proposition. Let $G, H, N$, and $Q$ be as in the above Lemma. Further, let $P$ be a prime ideal of $k G$ with $P \supset Q^{G}$. Then the following are equivalent:

i. $P$ is minimal over $Q^{G}$;

ii. $P \cap k N=Q^{G} \cap k N$;

iii. height $P=$ height $Q$.

Proof. (i $\Rightarrow$ ii) Put $I=Q^{G} \cap k N=\bigcap_{g \in G}(Q \cap k N)^{g}$, as in the Lemma (and its proof), and observe that $I$ is a $G$-prime ideal of $k N$. Indeed, as in (2), $Q \cap k N=$ $\bigcap_{h \in H} Q_{0}^{n}$ for some prime $Q_{0}$ of $k N$, and so $I=\bigcap_{g \in G}(Q \cap k N)^{g}=\bigcap_{g \in G} Q_{0}^{g}$. Clearly, $I \subset P \cap k N$. Suppose, by way of contradiction, that this inclusion is strict. Then, by [13, Lemma 3.4(i)], there exists an element $c \in P \cap k N$ such that $c$ is regular modulo $I$. Since $k G / I k G$ is free as left and right $k N / I$-module, $c$ is also regular in $k G$ modulo $I k G$. Therefore, $\operatorname{Kdim}(k G / I k G+c k G)_{k G}<$ $\operatorname{Kdim}(k G / I k G)$; see $[4,13.6]$. Further, by the above Lemma, $\operatorname{Kdim}(k G / I k G)=$ $\operatorname{Kdim}\left(k G / Q^{G}\right)$. Since $I k G+c k G \subset Q^{G}+c k G$, we obtain altogether that

$$
\operatorname{Kdim}\left(k G / Q^{G}+c k G\right)_{k G}<\operatorname{Kdim}\left(k G / Q^{G}\right) .
$$

By the above Lemma again, we know that $k G / Q^{G}$ is homogeneous. So the last inequality forces $c$ to be regular modulo $Q^{G}$ in $k G$; see (1.4 ii). Inasmuch as 
$c \in P$, we deduce from $[4,10.8]$ that $P$ cannot be minimal over $Q^{G}$, contrary to our hypothesis.

(ii $\Rightarrow$ iii) Recall from the previous part of the proof that $Q \cap k N=\bigcap_{h \in H} Q_{0}^{n}$ for some prime $Q_{0}$ of $k N$, and $Q^{G} \cap k N=\bigcap_{g \in G} Q_{0}^{g}$. Therefore, (ii) implies that $P \cap k N=\bigcap_{g \in G} Q_{0}^{g}$. By [11, Lemma 2.5(i)], we conclude that height $Q=$ height $Q_{0}=$ height $P$, thereby proving (iii).

(iii $\Rightarrow$ i) Assume height $Q=$ height $P$. If $P$ is not minimal over $Q^{G}$ then $P$ contains a prime, $P^{\prime}$, that is minimal over $Q^{G}$. Since we already have seen that (i) implies (iii), we know that height $P^{\prime}=$ height $Q$ and, consequently, height $P=$ height $P^{\prime}$. But this last equality is impossible, as $P$ properly contains $P^{\prime}$. The proof of the proposition is now complete.

\section{The proof}

In this section, $G$ will always denote a polycyclic-by-finite group. Thus, the group algebra $k G$ is left and right noetherian and satisfies the strong second layer condition.

3.1. The Roseblade subgroup. Following Roseblade [15], a subgroup $H$ of $G$ is called orbital if $H$ has only finitely many $G$-conjugates. An orbital subgroup $H$ is isolated if there is no orbital subgroup $H^{\prime}$ of $G$ with $H \subsetneq H^{\prime}$ and $\left[H^{\prime}: H\right]$ finite. Finally, a polycyclic-by-finite group is said to be orbitally sound if all its isolated orbital subgroups are normal. As in [15], we shall write

$$
\operatorname{nio}(G)
$$

for the intersection of the normalizers of all isolated orbital subgroups of $G$. The crucial properties of $\operatorname{nio}(G)$ for our purposes are the following:

- $\operatorname{nio}(G)$ is an orbitally sound normal subgroup of finite index in $G$. Moreover, $\operatorname{nio}(G)$ is the unique maximal such subgroup and nio $(G)$ contains every finite-by-nilpotent normal subgroup of $G$ (see $[15,1.3]$ ).

- The group algebra of $\operatorname{nio}(G)$ is catenary [15, Corrigenda].

Since images of orbitally sound groups are clearly orbitally sound as well, the maximality property of nio $(G)$ stated above implies in particular that, for every epimorphism $\varphi: G \rightarrow \bar{G}$, one has $\varphi(\operatorname{nio}(G)) \subset \operatorname{nio}(\bar{G})$.

The proof of the following lemma assumes some familiarity with [11], [10].

Lemma. Assume that $G \neq \operatorname{nio}(G)$. Then every faithful prime ideal of $k G$ is induced from some proper subgroup of $G$ containing $\operatorname{nio}(G)$.

Proof. Let $P$ be the prime in question, and let $H$ denote the normalizer of its vertex. Then $H$ contains nio $(G)$ and $P$ is induced from $k H$; see [10, (2.2) and Lemma 2.5]. If $H \neq G$ we are done; so assume that $H=G$. In this case, [10, Lemma 2.3] implies that $P$ is induced from $k \Delta$, where $\Delta=\Delta(G)$ denotes the FC-center of $G$. Since $\Delta$ is finite-by-abelian, $\Delta$ is contained in $\operatorname{nio}(G)$. Therefore, 
by transitivity of induction [11, Lemma 1.2(iii)], $P$ is induced from $k[\operatorname{nio}(G)]$ as well and the proof is complete.

3.2. A special case. The following Lemma proves the requisite condition (1) in the special case where the lower prime is induced from a subgroup of finite index in $G$ whose group algebra is catenary.

Lemma. Let $H$ be a subgroup of finite index in $G$ such that $k H$ is catenary. Then, for any pair $P \subsetneq P^{\prime}$ of adjacent primes of $k G$ such that $P$ is induced from $k H$, one has height $P^{\prime}=$ height $P+1$.

Proof. Write $P=Q^{G}$ for some ideal $Q$ of $k H$ which we may assume prime, by Lemma 2.2. By Proposition 2.3, height $Q=$ height $P$. Choose an ideal $Q^{\prime}$ of $k H$ containing $Q$ and maximal with respect to $\left(Q^{\prime}\right)^{G} \subset P^{\prime}$. Then, by $(2.2), Q^{\prime}$ is prime and $P^{\prime}$ is minimal over $\left(Q^{\prime}\right)^{G}$. Moreover, Proposition 2.3 gives height $Q^{\prime}=$ height $P^{\prime}$. Therefore, it suffices to show that height $Q^{\prime}=$ height $Q+1$ or, since $k H$ is catenary, that $Q$ and $Q^{\prime}$ are adjacent. Suppose otherwise and fix a prime $I$ of $k H$ properly between $Q$ and $Q^{\prime}$. Then height $Q<$ height $I<$ height $Q^{\prime}$. Further, $P=Q^{G} \subset I^{G} \subset\left(Q^{\prime}\right)^{G} \subset P^{\prime}$, and so $P^{\prime}$ contains a prime, $J$, of $k G$ minimal over $I^{G}$. By Proposition 2.3 again, height $J=$ height $I$. Consequently, all inclusions in $P=Q^{G} \subset J \subset P^{\prime}$ are strict, contradicting adjacency of $P$ and $P^{\prime}$.

3.3. Proof of the Theorem. To show that $k G$ is catenary, we argue by induction on the index $[G: \operatorname{nio}(G)]$. The case $G=\operatorname{nio}(G)$ is known [15]. So assume $G \neq \operatorname{nio}(G)$, and let $P \subsetneq P^{\prime}$ be adjacent primes of $k G$. We have to show that height $P^{\prime}=$ height $P+1$. In view of Lemma 1.2, we may pass to $G / P^{\dagger}$ and thus reduce to the case where $P$ is faithful. Note also that $[G: \operatorname{nio}(G)] \geq\left[G / P^{\dagger}: \operatorname{nio}\left(G / P^{\dagger}\right)\right]$; see $(3.1)$. Thus, by Lemma $3.1, P$ is induced from $k H$ for some proper subgroup $H$ of $G$ containing nio $(G)$. Since $\operatorname{nio}(G)$ is contained in $\operatorname{nio}(H)$, by the maximality property of nio, we have $[H: \operatorname{nio}(H)]<[G: \operatorname{nio}(G)]$ and so $k H$ is catenary, by induction. Lemma 3.2 now completes the proof.

\section{Concluding remarks}

$G$ will continue to denote a polycyclic-by-finite group.

Roseblade's work [15] establishes catenarity of $k G$ up to a finite index: $G_{0}=$ $\operatorname{nio}(G)$ is a normal subgroup of finite index in $G$ such that $k G_{0}$ is catenary. Putting $R=k G_{0}$ and $S=k G$, we have

$$
S=R * \Gamma,
$$

a crossed product of the finite group $\Gamma=G / G_{0}$ over $R$. It is tempting to approach the catenary problem for $k G$ from a more general angle by establishing a positive answer to the following: 
Question. Suppose that $S=R * \Gamma$ is a crossed product with $R$ an affine noetherian k-algebra and $\Gamma$ a finite group. If $R$ satisfies (1), will $S$ do so as well?

Recall that (without any hypotheses on $R$ ) the ideals of $R$ of the form $P \cap R$, for some prime ideal $P$ of $S=R * \Gamma$, are precisely the $\Gamma$-primes of $R$ and that these ideals are exactly those of the form $Q_{\Gamma}=\bigcap_{x \in \Gamma} Q^{x}$ for some prime $Q$ of $R$. Moreover, if $Q_{\Gamma} \subset Q_{\Gamma}^{\prime}$ then we may assume, after replacing $Q$ by a suitable $\Gamma$-conjugate, that $Q \subset Q^{\prime}$. The latter will be adjacent if $Q_{\Gamma}$ and $Q_{\Gamma}^{\prime}$ are adjacent $\Gamma$-primes. Finally, $P \cap R=Q_{\Gamma}$ entails height $P=$ height $Q$. See [12] for details. A positive answer to the above question would therefore result if the following were true:

Question'. Do adjacent primes of $S=R * \Gamma$ contract to adjacent $\Gamma$-primes of $R$ ?

Here is what appears to be known about Question':

- Without noetherian hypotheses, the answer is negative in general. Indeed, Kaplansky [6] constructs an extension of commutative (non-noetherian) rings $R \subset S$, with $S=R * \Gamma$ and $\Gamma$ the group of order 2, so that Question' fails for $S$.

- Question' has a positive answer if $R$ is affine PI. This is an easy application of Schelter's catenarity result [16, Theorem 4] for affine PI-algebras.

It would be interesting to have more definitive results about this topic.

\section{Acknowledgment}

The authors would like to thank K. A. Brown for reading a preliminary draft of this article and for his valuable comments. We also would like to thank L. W. Small for his helpful remarks concerning $§ 4$.

\section{References}

[1] K.A. Brown, The structure of modules over polycyclic groups, Math. Proc. Cambridge Philos. Soc. 89 (1981), 257-283.

[2] O. Gabber, Équidimensionalité de la variété caractéristique, exposé de O. Gabber rédigé par T. Levasseur, Université de Paris VI, 1982.

[3] K.R. Goodearl and T.H. Lenagan, Catenarity in quantum algebras, J. Pure Appl. Algebra 111 (1996), 123-142.

[4] K.R. Goodearl and R.B. Warfield, Jr., An introduction to noncommutative Noetherian rings, London Mathematical Society Student Texts, 16., Cambridge University Press, Cambridge, 1989.

[5] A.V. Jategaonkar Localization in Noetherian rings, London Mathematical Society Lecture Note Series, 98., Cambridge University Press, Cambridge-New York, 1986.

[6] I. Kaplansky, Adjacent prime ideals, J. Algebra 20 (1972), 94-97.

[7] B. Lemonnier, Dimension de Krull et codéviation, Application au théorème d'Eakin, Comm. Algebra 6 (1978), 1647-1665.

[8] T.H. Lenagan, Enveloping algebras of solvable Lie superalgebras are catenary, Contemp. Math. 130 (1992), 231-236.

[9] E.S. Letzter, Prime ideals in finite extensions of Noetherian rings, J. Algebra 135 (1990), 412-439. 
[10] M. Lorenz, Prime ideals in group algebras of polycyclic-by-finite groups: vertices and sources, report on joint work with D. S. Passman, in: Séminaire d'Algèbre Paul Dubreil et Marie-Paule Malliavin, Lect. Notes in Mathematics No. 867, pp. 406-420, SpringerVerlag, Berlin-Heidelberg, 1981.

[11] M. Lorenz and D. S. Passman, Prime ideals in group algebras of polycyclic-by-finite groups, Proc. London Math. Soc. (3) 43 (1981), 520-543.

[12] _ Prime ideals in crossed products of finite groups, Israel J. Math. 33 (1979), 89-132; Addendum, Israel J. Math. 35 (1980), 311-322.

[13] Centers and prime ideals in group algebras of polycyclic-by-finite groups, J. Algebra 57 (1979), 355-386.

[14] J.C. McConnell and J.C. Robson, Noncommutative Noetherian rings, Pure and Applied Mathematics., John Wiley \& Sons, Ltd., Chichester, 1987.

[15] J. E. Roseblade, Prime ideals in group rings of polycyclic groups, Proc. London Math. Soc. (3) 36 (1978), 385-447; Corrigenda, Proc. London Math. Soc. (3) 38 (1979), 216218.

[16] W. Schelter, Non-commutative affine P.I. rings are catenary, J. Algebra 51 (1978), 1218.

[17] D. Segal, unpublished manuscript, ca. 1976.

[18] P.F. Smith, On the dimension of group rings, Proc. London Math. Soc. 25 (1972), 288302.

[19] J.T. Stafford, On the regular elements of Noetherian rings, in: Ring Theory, Proceedings of the 1978 Antwerp Conference, (F. Van Oystaeyen, Ed.), pp. 257-277, Lecture Notes in Pure and Appl. Math., 51, Dekker, New York, 1979.

[20] A.E. Zalesskii, Irreducible representations of finitely generated nilpotent torsion-free groups, Math. Notes 9 (1971), 117-123.

Department of Mathematics, Texas A\&M University, College Station, TX 77843

E-mail address: letzter@math.tamu.edu

Department of Mathematics, Temple University, Philadelphia, PA 19122-6094

E-mail address: lorenz@math.temple.edu 